\title{
The validity of axisymmetric assumptions when investigating pulsatile biological flows
}

\author{
$\begin{array}{lll}\text { R. A. Jamison } & \text { G. J. Sheard } \\ & \text { K. Ryan } & \end{array}$ \\ A. Fouras ${ }^{4}$
}

(Received 15 August 2008; revised 6 January 2009)

\begin{abstract}
Computational fluid simulations of biological flows is increasingly popular due to its inexpense and ability to define the flow throughout the entire domain-both common limiting factors for experimental work. A common assumption has been that both the geometry and the flow field through an aneurysm is axisymmetric; however, investigations into non-biological flows have seen that even with an axisymmetric geometry, non-axisymmetric flow may develop. Idealised geometries are used to investigate these biological flows as it simplifies the model to enable an improved understanding of the effect geometry has on the flow. Additionally this simplification allows the implementation of a computationally cheaper axisymmetric code. We test this axisymmetric assumption by applying Floquet stability analysis to investigate the stability of the flow and thus determine when an axisymmetric aneurysmal flow is unstable to non-axisymmetric instabilities. Dimensions of the model are selected to be consistent with a high risk
\end{abstract}

http://anziamj . austms.org.au/ojs/index.php/ANZIAMJ/article/view/1460 gives this article, (c) Austral. Mathematical Soc. 2009. Published January 22, 2009. ISSN 1446-8735. (Print two pages per sheet of paper.) 
aneurysm in the human abdominal aorta and Reynolds numbers relevant to aneurysms in large arteries are examined. The presence of three dimensional instabilities has a significant impact on the validity of the assumption of axisymmetry. The maximum streamwise vorticity in the perturbation fields is found to occur at the downstream section of the aneurysm, implying that it is in these areas that the results of axisymmetric simulations differ the most from fully three dimensional flow.

\section{Contents}

1 Introduction

2 Method

3 Results

4 Discussion

5 Conclusion

References

\section{Introduction}

An aneurysm is a localised, blood filled dilation of a blood vessel caused by disease or weakening of the vessel wall [12]. This study investigates the most common fusiform aneurysm, the Abdominal Aortic Aneurysm (AAA), which is usually located upstream of a major abdominal bifurcation [8].

Rupture of an aneurysm occurs when the vessel wall becomes too weak and the stress within the aneurysm exceeds the tensile strength of the wall [13]. 
Almost $90 \%$ of ruptures lead to death [4]. At present there is no reliable basis on which to evaluate susceptibility to rupture of an AAA. Medical intervention requires the risk of rupture to be weighed against operation morbidity [8], with surgical treatment recommended for AAAs exceeding $5 \mathrm{~cm}$ in maximal diameter or with expansion rates $>0.5 \mathrm{~cm} /$ year [2]. This method for determining the need for medical intervention is crude as it does not take into account the geometry of the aneurysm - a major determining factor of the fluid stresses acting on the aneurysms walls.

Research into aneurysms using computational fluid dynamics (CFD) commonly simplifies the geometry and the symmetry of the flow to reduce the complexity of the modeled system. For example, Cowling et al. [3], Salsac et al. [9] and Sheard et al. [10] considered an axisymmetric geometry, with the numerical simulations of Sheard et al. [10] considering only axisymmetric flow. While Cowling et al. [3], Salsac et al. [9] and Lasheras [8] observed possible flow asymmetry in axisymmetric fusiform aneurysms, no study has yet to investigate the onset of this non-axisymmetric flow. Neglecting to take this three dimensionality into account in previous studies limits the application of their findings.

This study aims to determine the stability of the flow in an axisymmetric fusiform aneurysm to non-axisymmetric perturbations and thus evaluate the axisymmetric assumption made by studies when computing aneurysmal flows. A range of vessel sizes and pulsatile flow conditions mimic conditions experienced by physical blood flows.

\section{Method}

The common layout shown in Figure 1 displays the geometry of the aneurysm: $\mathrm{U}$ is defined as the time averaged mean flow velocity; $\mathrm{D}$ is the undilated blood vessel diameter; $R_{c}=R / D$ is the normalised radius of curvature between the blood vessel and the aneurysmal bulge; and $L$ and $W$ are the length and width 


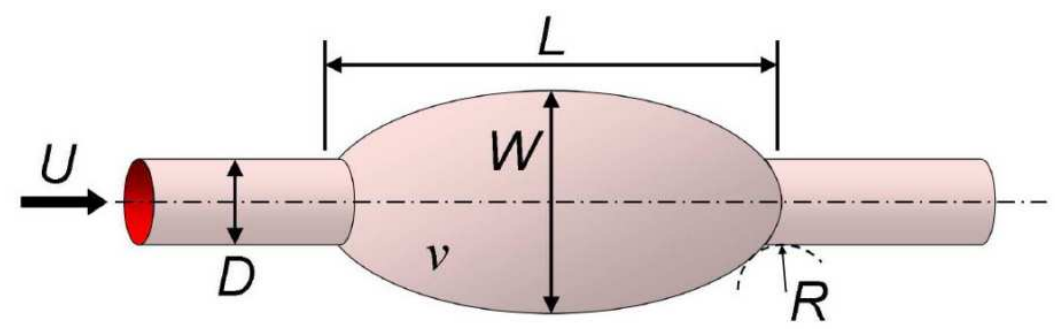

Figure 1: Common idealised aneurysm with major dimensions [10].

of the bulge, respectively. The model geometry used in this study represents typical abdominal aortic aneurysms, with the geometry parameters of $\mathrm{L} / \mathrm{D}=$ $4, \mathrm{~L} / \mathrm{W}=1.76, \mathrm{R}_{\mathrm{c}}=0.5$; the upstream and downstream artery segments are each 3D long to ensure accurate flow development prior to the aneurismal bulge. To enable direct comparisons to be made with previous research, the periodic time history of blood flow is kept consistent with previous research. The amplitude of the inlet velocity is $\mathrm{U} / 2$ [10] with the periodic blood flow approximated using the sinusoidal function

$$
\frac{u_{\text {inlet }}}{\mathrm{u}}=u_{\text {profile }} \times\left(1+\frac{1}{2} \sin \omega t\right),
$$

where

$$
u_{\text {profile }}=8 \times\left(0.25-y^{2}\right),
$$

with $v_{\text {inlet }}$ and $w_{\text {inlet }}$ set to zero; $y$ is the nondimensional distance from the axis of rotation at the inlet, with $y=1 / 2$ corresponding to the wall. This simplification is used for fundamental flow investigations as an approximate signal reduces the number of parameters, enabling the effect of geometry to be more thoroughly examined. A parabolic profile is used for the inflow condition, which is a simplification of the Womersley solution for periodic pipe flows. The flow is found to adapt to a Womersley profile [14] in the upstream segment, well before the aneurysmal bulge. The initial flow field is $u_{\text {inlet }}$ applied along the central core of the geometry, with zero velocities in the outer region of the aneurysm bulge. 
For pulsatile circulatory flow, two dimensionless parameters characterise the flow conditions. The Reynolds number is defined as

$$
\mathrm{Re}=\frac{\mathrm{UD}}{v},
$$

where $v$ is the kinematic viscosity (for whole blood $v=2.547 \times 10^{-6} \mathrm{~m}^{2} \mathrm{~s}^{-1}$ [10]). The Reynolds number was varied from 100 to 2000, to account for the various sizes and locations of aneurysms. The Reynolds number range chosen corresponds to blood vessels ranging over approximately $1-20 \mathrm{~mm}$, which not only covers the human abdominal aorta (generally $15-20 \mathrm{~mm}[4]$ ), but also smaller blood vessels found elsewhere in the body. For arteries greater than $0.5 \mathrm{~mm}$ in diameter, the non-Newtonian and multi-phase properties of blood can be safely ignored [7]. In these simulations blood is also assumed to be incompressible and homogeneous.

The second dimensionless parameter, the Womersley number, is

$$
\alpha=\frac{\mathrm{D}}{2} \sqrt{\frac{\omega}{v}},
$$

where $\omega$ is the frequency of the pulsatile flow. The bulk of this study considers a constant Womersley number typical of resting heart conditions. For reference, $\alpha=16.9$ corresponds to a heart rate of 70 beats per minute if $\mathrm{D}=20 \mathrm{~mm}$ and $v=2.547 \times 10^{-6} \mathrm{~m}^{2} \mathrm{~s}^{-1}$.

The numerical simulations were performed using an in-house spectral element software package which solves the incompressible time dependant NavierStokes equations $[5,11]$. Within each element, polynomial shape functions are employed, and high spatial convergence is achieved by the use of efficient Gauss-Legendre-Lobatto quadrature. Spatial resolution is controlled by varying the polynomial degree, $\mathrm{N}$, within each element. Higher resolution was used at the walls and around the aneurysmal bulge as this is the main area of interest and the area that the largest variable gradients are likely to occur. The inlet boundary was set as a transient Dirichlet boundary condition with the previously defined inlet velocity. Additionally, a high order 
Neumann boundary condition is imposed on the outward normal gradient of the pressure field [6]. The outlet boundary was set as a Dirichlet boundary condition with the pressure at the boundary set to zero to act as a reference pressure. Furthermore zero normal gradient of velocity is naturally imposed as a consequence of the treatment the Galerkin solution of the diffusion term.

A grid independence study determined the mesh density required to obtain the desired accuracy. The highest Reynolds number investigated, $R e=2000$, was used as the test case as higher Reynolds number flows are more sensitive to resolution deficiencies in simulations. An $\mathrm{L}_{2}$ norm of the velocity was taken periodically, with the period being equal to that of the inflow. The $\mathrm{L}_{2}$ norm is the integral of the velocity magnitude over the domain:

$$
\mathrm{L}_{2} \text { norm }=\int_{\Omega}|\mathrm{U}| \mathrm{d} \Omega,
$$

where $\Omega$ is the computational domain and $|\mathrm{U}|$ is the velocity magnitude. The $L_{2}$ norm of each periodic flow was compared to the highest resolution case, providing an estimate of the percentage uncertainty of each case. These results are plotted in Figure 2 for element polynomial degree $\mathrm{N}=4, \ldots, 8$. We concluded that for polynomials above degree seven, there was a negligible gain in accuracy. Hence elements of degree seven were used thereafter.

In order to determine if three dimensional features are present in the flow, a linear stability analysis technique was used. Floquet stability analysis provides a prediction of the three dimensional instabilities in a steady or periodic axisymmetric flow $[1,11]$. This is achieved by inspecting the evolution of a small three dimensional perturbation to a two dimensional base flow. Floquet analysis returns an eigenvalue representing the magnitude of the Floquet multiplier $|\mu|$, which in turn represents an amplification factor applied to the three dimensional perturbation between successive periods. If $|\mu| \geq 1$, the perturbation will grow over time and the solution is unstable. If $|\mu|<1$, the perturbation will decay and the axisymmetric flow is stable. The perturbations are periodic in the azimuthal direction, with azimuthal wavelength $2 \pi / m$, where $m$ is the mode number. The perturbation fields 


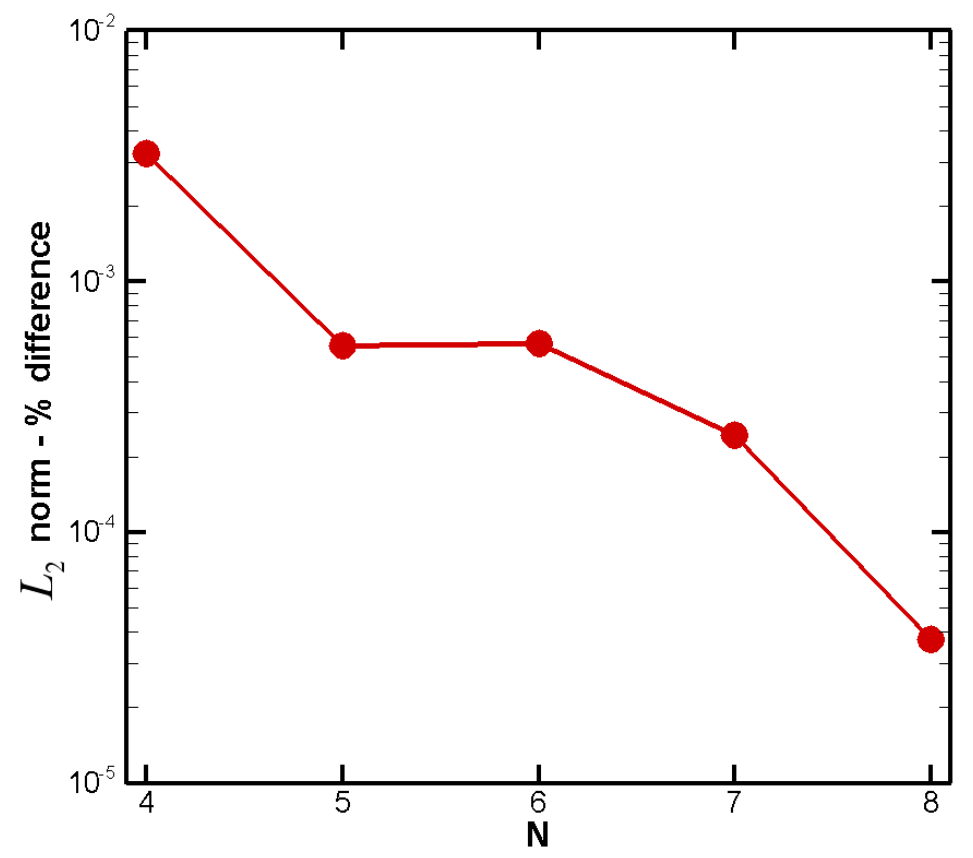

Figure 2: Percentage difference in $\mathrm{L}_{2}$ norm of the velocity versus element polynomial degree N. Simulations conducted with $\operatorname{Re}=2000$ and $\alpha=16.9$. 
are solved using the linearised Navier-Stokes equations, which decouples each individual azimuthal mode of the three dimensional perturbation. This permits us to analyse the stability of individual wavelengths in isolation, thus greatly reducing the computational expense. As we only consider the growth when the perturbation is small, the non-linear components are negligible [1].

Several azimuthal wavelengths were investigated in order to determine the wavelength that would dominate the three dimensional characteristics of the flow. However, axisymmetry imposes a restriction whereby only integer modes are available. The first four modes were investigated, representing wavelengths of $2 \pi, \pi, 2 \pi / 3$ and $\pi / 2$, as studies have shown that these modes are consistently more unstable than higher mode numbers $[9,10]$, which are inevitably damped by viscous effects.

\section{$3 \quad$ Results}

Figure 3 displays the Floquet multiplier in terms of the azimuthal wavelength for the idealised axisymmetric geometry investigated, for $500 \leq \mathrm{Re} \leq 750$. Figure 3 suggests an approximation of both the Reynolds number and azimuthal wavelength for which transition to three dimensional flow occurs. The largest Floquet multipliers near the transition were found to occur at mode number $m=2$, corresponding to an azimuthal wavelength of $\pi$.

A prediction of the exact Reynolds number for which the transition will occur was made by plotting the four data points for the mode number $\mathrm{m}=2$ that are closest to $|\mu|=1$. To this a parabola was fitted to the data and the equation it yielded solved for $|\mu|=1$. This process predicted a critical Reynolds number of $\operatorname{Re}_{\text {crit }}=610.68$.

Figure 4 displays the isosurfaces of the streamwise perturbation vorticity for the dominant mode number $(m=2)$ at $R e=\operatorname{Re}_{\text {crit }}$ for a complete cardiac cycle with a $1 / 4$ period progression. Figures $4 b$ and $4 d$ represent peak systole and diastole, respectively, at the input of the domain. The direction 


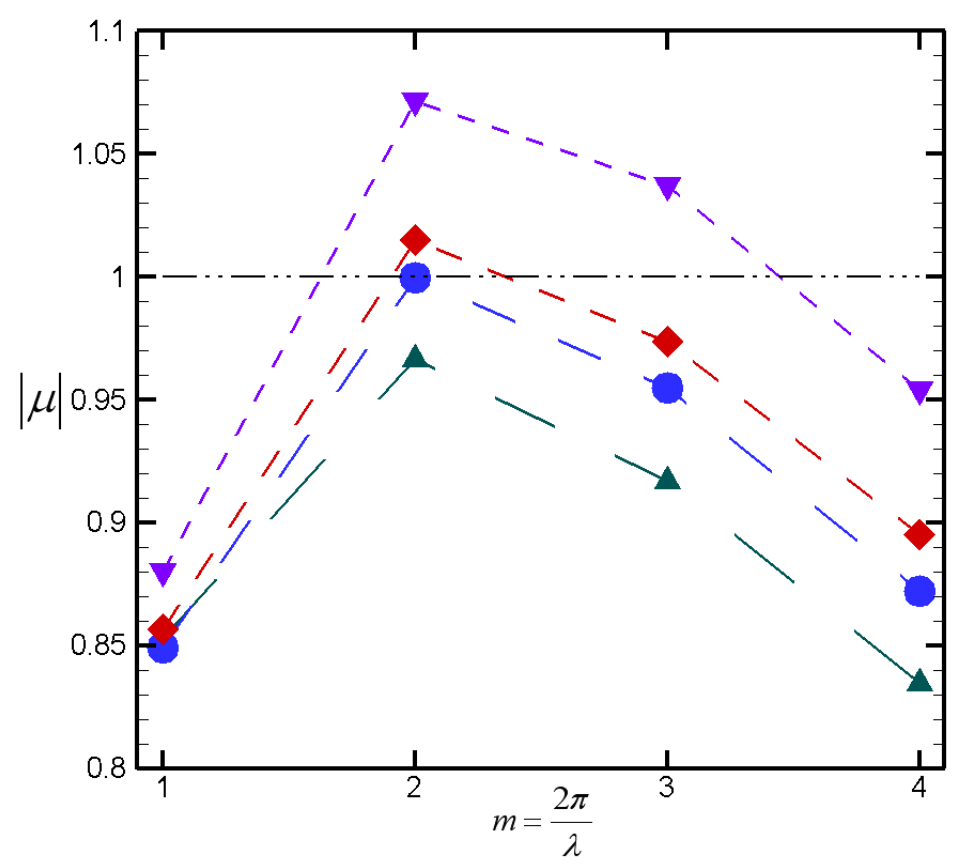

Figure 3: : Floquet multiplier plotted against mode number $m$ for $500 \leq$ $\operatorname{Re} \leq 750$ and $\alpha=16.9$. The dash-dot line represents the stability threshold: $\Delta \operatorname{Re}=500 ; \bullet \operatorname{Re}=610 ; \diamond \operatorname{Re}=650$; and $\boldsymbol{\nabla} \operatorname{Re}=750$. 

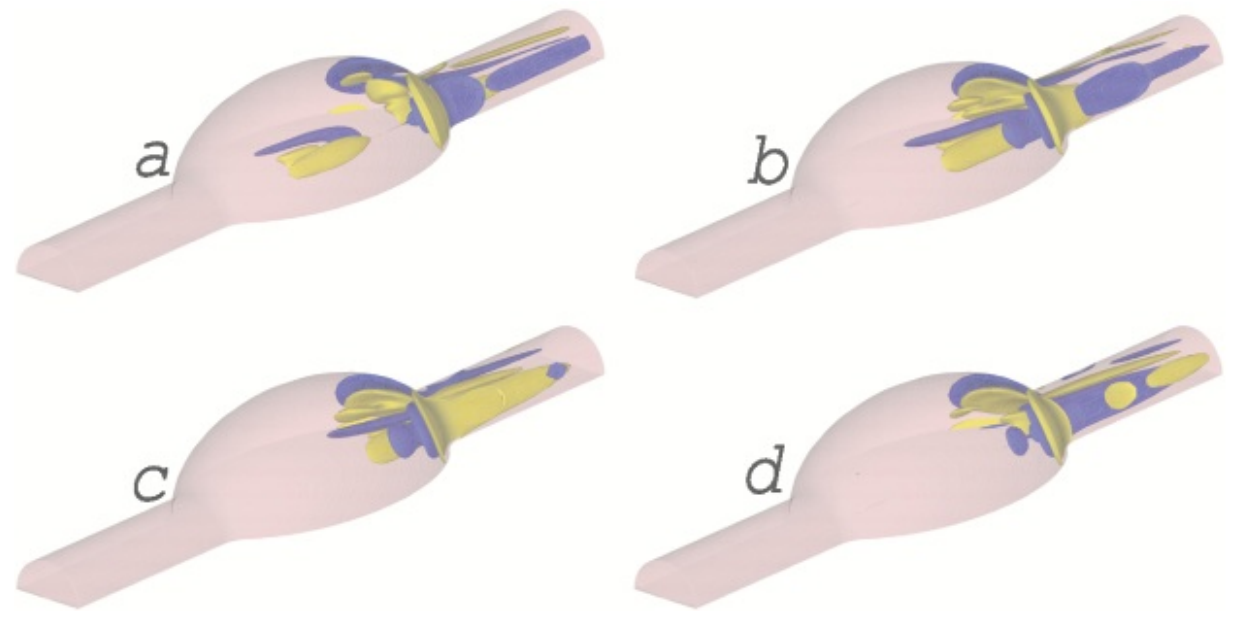

FigURE 4: Isosurfaces of the streamwise perturbation vorticity for mode number $m=2$ with $R e=R e_{\text {crit }}$. Each figure represents a snapshot of the mode development taken at $1 / 4$ period progression. Blue represents negative vorticity and yellow represents positive vorticity. Flow is from the bottom left to the top right.

of flow is from the bottom left to the top right of each image. These are shown for only one half of the axisymmetric geometry as the mode number $m=2$ perturbation is repeated twice over the computational domain. The streamwise perturbation vorticity is seen to occur in two main regions; at the wall downstream of the bulge, Figure $4 c$, and in the centre of the bulge, Figure 4a. The streamwise vorticity in the centre of the bulge is due to the vortex ring that is created as separation occurs when peak systole progresses into the aneurysm. Although the vorticity is seen to vary substantially over each cardiac cycle, the peak vorticity of the $m=2$ instability consistently occurs at the downstream section of the aneurismal bulge at the vessel wall.

Additionally, for $R e<\operatorname{Re}_{\text {crit }}$ the majority of the flow was seen to remain constrained to the central core of the aneurysm, with little comparative flow within the aneurysmal bulge. It is this behaviour that helps establish the 
presence of axisymmetric flow at these lower Reynolds numbers.

Figure 5 shows the Floquet multiplier in terms of the azimuthal wavelength with $\operatorname{Re}=750$ for $12 \leq \alpha \leq 23.6$. As $\alpha$ was increased (representing increasing the heart rate) smaller Floquet multipliers were returned. From this observation when investigating higher Womersley numbers the Reynolds number range for which flow will be axisymmetric will be greater than that for lower heart rates.

\section{Discussion}

The calculation of $\operatorname{Re}_{\text {crit }}$, the Reynolds number at which the onset of three dimensional flow will begin, enables the diameters of blood vessels for which axisymmetric simulations are appropriate to be determined. This study predicts that for $\mathrm{Re}<610$ (corresponding to a blood vessel diameter $\mathrm{D}=$ $6.2 \mathrm{~mm}$, for $v=2.547 \times 10^{-6} \mathrm{~m}^{2} \mathrm{~s}^{-1}, \mathrm{U}=0.25 \mathrm{~ms}^{-1}$ and $\alpha=16.9$ ) the flow will be axisymmetric for this particular geometry. It follows that simulations of flows with $\operatorname{Re}<610$ are able to be performed under the assumption of axisymmetry. However, as most research in this field is into AAAs, where the blood vessel is the aorta $(15-20 \mathrm{~mm}$ in diameter, relating to the Reynolds number range $1500 \leq \operatorname{Re} \leq 2000$, for $v=2.547 \times 10^{-6} \mathrm{~m}^{2} \mathrm{~s}^{-1}$ ), it is unlikely that realistic Reynolds number flow will be axisymmetric.

Three dimensional structures in the flow develop most strongly in the downstream section of the aneurysm, close to the wall. This finding implies that three dimensional effects will alter the velocities of previous studies, and thus change derived values such as wall shear stress. However, it is yet to be determined how substantial the effect of this three dimensionality is, and thus whether the variation in computed results is significant. Future work will use fully 3D simulations to enable an accurate comparison to be made to determine the extent of this three dimensionality.

A second consideration is the Womersley number. If low Womersley num- 


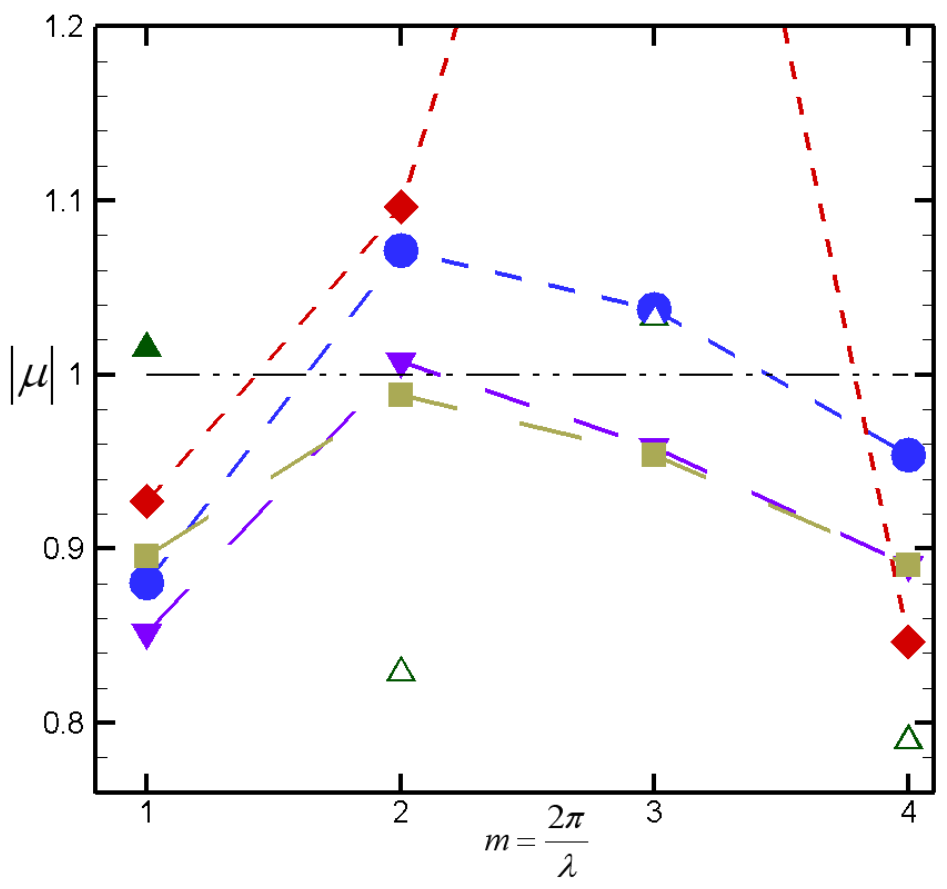

FigURE 5: Floquet multiplier plotted against mode number $\mathfrak{m}$ for $12 \leq \alpha \leq$ 23.6 and $\mathrm{Re}=750$. The dash-dot line represents the stability threshold: $\checkmark \alpha=12.0 ; \boldsymbol{\Delta} \alpha=15.0 ; \bullet \alpha=16.9 ; \boldsymbol{\nabla} \alpha=19.8$; and $\mathbf{\square} \alpha=23.6$. Filled and hollow symbols represent regular and quasi-periodic Floquet multipliers respectively. 
bers are being considered, the range of Reynolds numbers available to be investigated will be reduced as non-axisymmetric flow will develop at lower Reynolds numbers.

\section{Conclusion}

This study predicts the critical Reynolds number for transition to three dimensional flow to be $\mathrm{Re}=610$ for the idealised geometry investigated, with the transition found to occur with mode number $m=2$. The maximum streamwise vorticity in the perturbation field was found near the downstream section of the aneurysmal bulge, suggesting that non-axisymmetric structures develop in this region. A strong correlation has been found between inflow frequency and the critical Reynolds number, with an increase of the inflow frequency resulting in a decrease of the critical Reynolds number.

Acknowledgements The computational resources of the Australian Partnership for Advanced Computing (APAC) were utilised to complete this study, thanks to a time allocation under the Merit Allocation Scheme.

\section{References}

[1] Barkley, D. \& Henderson, R. D., Three-dimensional Floquet stability analysis of the wake of a circular cylinder. J. Fluid Mech. 322 (1996), 215-241. doi:10.1017/S0022112096002777 C718, C720

[2] Brown, P. M., Zelt D. T., Sobolev B., The risk of rupture in untreated aneurysms: The impact of size, gender, and expansion rate. J. Vasc. Surg. 37 (2003), 280-284. doi:10.1067/mva.2003.119 C715 
[3] Cowling R., Soria J., Flow Visualisation through Model Abdominal Aortic Aneurysm, Fourth Australian Conference on Laser Diagnostics in Fluid Mechanics and Combustion, The University of Adelaide, South Australia, Australia, 7-9 December 2005, 33-36. C715

[4] Egelhoff C. J., Budwig R. S., Elger D. F., Khraishi T. A., Model studies of the flow in abdominal aortic aneurysms during resting and exercise conditions. J. Biomech, 32 (1999), 1319-1329. doi:10.1016/S0021-9290(99)00134-7 C715, C717

[5] Karniadakis, G. E. \& Triantafyllou, G. S., Frequency selection and asymptotic states in laminar wakes. J. Fluid Mech. 199 (1989), 441-469. doi:10.1017/S0022112089000431 C717

[6] Karniadakis, G. E., Israeli, M. \& Orszag, S. A., High-order splitting methods for the incompressible Navier-Stokes equations, J. Comp. Phys. 97 (1991), 414-443. doi:10.1016/0021-9991(91)90007-8 C718

[7] Ku, D. N., Blood flow in arteries, Annual review of Fluid Mechanics, 29 (1997), 399-434. doi:10.1146/annurev.fluid.29.1.399 C717

[8] Lasheras J., The Biomechanics of Arterial Aneurysms, Annual Review of Fluid Mechanics, 39 (2007), 293-319 doi:10.1146/annurev.fluid.39.050905.110128 C714, C715

[9] Salsac, A. V., Sparks, S. R., Chomaz, J. M. \& Lasheras, J. C., Evolution of the wall shear stresses during the progressive enlargement of symmetric abdominal aortic aneurysms, J. Fluid Mech., 560 (2006), 19-51. doi:10.1017/S002211200600036X C715, C720

[10] Sheard, G. J., Evans, R. G., Denton, K. M. \& Hourigan, K., Undesirable Haemodynamics in Aneurysms, In Proceedings of the IUTAM Symposium on Unsteady Separated Flows and Their Control, Hotel Corfu Chandris, Corfu, Greece, 18-22 June 2007 C715, C716, C717, C720 
[11] Sheard, G. J. \& Ryan, K., Pressure-driven flow past spheres moving in a circular tube, J. Fluid Mech. 592 (2007), 233-262. doi:10.1017/S0022112007008543 C717, C718

[12] Stedman, 2002, The American Heritage Stedmans Medical Dictionary, Houghton Mifflin Company, Massachusetts. C714

[13] Steinman, D. A., Vorp, D. A. \& Ethier, C. R., Computational modelling of arterial biomechanics: Insights into pathogenesis and treatment of vascular disease, J. Vascular Surgery, 37 (2003), 1118-1128. doi:10.1067/mva.2003.122 C714

[14] Waite, L. \& Fine, J. (2007). Applied biofluid mechanics. United Stated of America: McGraw-Hill. C716

\section{Author addresses}

1. R. A. Jamison, Monash University Biomedical Engineering Technology Alliance, Div. Biological Engineering, Monash University, Victoria 3800, Australia; and Fluids Laboratory for Aeronautical \& Industrial Research, Dept. Mechanical and Aerospace Engineering, Monash University, Victoria 3800, Australia.

mailto:Greg. Sheard@eng. monash. edu . au

2. G. J. Sheard, Monash University Biomedical Engineering Technology Alliance, Div. Biological Engineering, Monash University, Victoria 3800, Australia; and Fluids Laboratory for Aeronautical \& Industrial Research, Dept. Mechanical and Aerospace Engineering, Monash University, Victoria 3800, Australia.

3. K. Ryan, Fluids Laboratory for Aeronautical \& Industrial Research, Dept. Mechanical and Aerospace Engineering, Monash University, Victoria 3800, Australia.

4. A. Fouras, Monash University Biomedical Engineering Technology 
Alliance, Div. Biological Engineering, Monash University, Victoria 3800, Australia; and Fluids Laboratory for Aeronautical \& Industrial Research, Dept. Mechanical and Aerospace Engineering, Monash University, Victoria 3800, Australia. 